\title{
Evaluation of methotrexate-conjugated gadolinium(III) for cancer diagnosis and treatment
}

This article was published in the following Dove Press journal:

Drug Design, Development and Therapy

\author{
Dan $\mathrm{Xu}$ \\ Shu-Ting Lu ${ }^{2}$ \\ Yu-Shuang $\mathrm{Li}^{2}$ \\ Aju Baidya ${ }^{2}$ \\ $\mathrm{Hao} \mathrm{Mei}^{2}$ \\ Yong $\mathrm{He}^{1}$ \\ $\mathrm{Bo} \mathrm{Wu}^{2}$
}

'Department of Nuclear Medicine, Zhongnan Hospital of Wuhan University, Wuhan University, Wuhan 43007I, PR China; ${ }^{2}$ Department of Radiology, Zhongnan Hospital of Wuhan University, Wuhan University Wuhan 43007I, PR China
Correspondence: Yong $\mathrm{He}$

Department of Nuclear Medicine,

Zhongnan Hospital of Wuhan University,

Wuhan University, 169 Donghu Road

street, Wuhan 43007I, Hubei province,

PR China

Tel +86 27 678I 2698

Email vincentheyong@163.com

Bo Wu

Department of Radiology, Zhongnan Hospital of Wuhan University, Wuhan University, Wuhan 43007I, PR China

Tel +8627 678I 2533

Email wubo5317@whu.edu.cn
Background: Gliomas are one of the most common types of primary brain tumors. It is usually evaluated by gadolinium(III)-based contrast agents by magnetic resonance imaging (MRI) in the clinic. Methotrexate (MTX), as a type of folate analog that inhibits the enzyme dihydrofolate reductase, is widely used as a chemotherapeutic agent to treat gliomas in the experiment.

Purpose: In this study, a novel theranostic agent MTX-DOTA-Gd (MTX-Gd) was synthesized, which integrates magnetic resonance imaging (MRI) with anticancer treatment.

Methods: MTX-Gd was synthesized by connecting MTX and Gd through 1,4,7,10-tetraazacyclododecane-1,4,7,10-tetraacetic acid (DOTA). The characterization of MTX-Gd was detected by ultraviolet (UV) and infrared spectroscopy (IR). To confirm the antitumor effect of MTX-Gd, the cytotoxicity of MTX-Gd was examined by the MTT assay. The contrast enhancement of the MTX-Gd was measured through MRI in vitro. Then, nude mice bearing C6 tumor xenografts were used to study in vivo imaging capabilities.

Results: The ultraviolet-visible-near infrared radiation (UV-NIR) absorption curve indicated that MTX-Gd had a broad absorption in the region of 500-700 nm. The formation of MTX-Gd was confirmed from the characteristic bands of MTX-DOTA-Gd in the $1413 \mathrm{~cm}^{-1}(\mathrm{C}-\mathrm{N}), 1577 \mathrm{~cm}^{-1}$ $\left(-\mathrm{NH}_{2}\right)$, and $3429 \mathrm{~cm}^{-1}(\mathrm{~N}-\mathrm{H})$, in the fourier-transform infrared (FTIR) spectra. MTX-Gd showed little difference in the cell viability compared with MTX, except for the highest concentration $(270 \mu \mathrm{M})$. In vitro, the imaging of MTX-Gd was significantly brighter than Gd-DOTA at the same concentration, and the brightness and signal intensity of MRI were increased followed by the increased concentration of MTX-Gd. And it also showed that MTX was not visualized on MRI. The other images revealed that the concentration of 4 mM MTX-Gd had the same imaging effect with the concentration of $10 \mathrm{mM}$ Gd-DOTA. Then, MTX-Gd was injected in nude mice bearing C6 tumor xenografts through the tail vein. Significant contrast enhancement was observed at the tumor site from $0.5 \mathrm{~h}$ to $3 \mathrm{~h}$. The signal of tumor area was strongest at $3 \mathrm{~h}$ due to accumulation by size effect of macromolecules.

Conclusion: A novel stable and unique theranostic agent (MTX-Gd) was successfully synthesized, and it has good stability, strong anticancer ability and excellent magnetic capacity. The methotrexate component of MTX-Gd, as a chemotherapeutic agent, played an important role in targeted therapies of cancer. The DOTA-Gd component of MTX-Gd performed as the MRI contrast agent. The superior MRI imaging performance and synergetic chemical antineoplastic ability of MTX-Gd was revealed, and it has great potential in the diagnosis and treatment of glioma and potentially other cancers, with prospects of clinical application in the near future.

Keywords: methotrexate, gadolinium, glioma, theranostics

\section{Introduction}

Methotrexate (MTX) is a folate analog that inhibits the enzyme dihydrofolate reductase. It is widely used to treat psoriasis, rheumatoid arthritis, and other autoimmune diseases. In addition, malignant diseases such as acute lymphoblastic leukemia (ALL), 
lymphoma, medulloblastoma, and osteosarcoma are treated with high doses of MTX or intrathecal injection of MTX.1,37-39 MTX at a dose of $\geq 1 \mathrm{~g} / \mathrm{m}^{2}$ (high-dose MTX, HDMTX) is the most efficient treatment against primary central nervous system lymphoma (PCNSL) and is the most widely used drug in prospective clinical trials. ${ }^{2,27-32}$ Numerous studies suggest that HDMTX $\left(1 \mathrm{~g} / \mathrm{m}^{2}\right)$ has a moderate blood-brain barrier (BBB) permeability (approximately $5 \%$ of plasma levels). ${ }^{3}$

Glioma is a common type of primary brain tumor that has high morbidity and mortality due to its location and locally invasive growth. Currently, gadolinium(III)-based contrast-enhanced magnetic resonance imaging (MRI) is a preferred choice for the clinical diagnosis of glioma and preoperative localization., ${ }^{1,33}$ Some studies have reported that MRI can make a good evaluation for the grading of gliomas. ${ }^{4,54-36}$ Measurement of relative cerebral blood volume (rCBV) can be used to improve the sensitivity of grading a tumor; the preoperative distinction between highgrade and low-grade gliomas showed that rCBV in intratumoral and peritumoral regions was significantly different. The combination of intratumoral and peritumoral rCBV provided overall better diagnostic accuracy and helped to reduce invasive interventions for nonsurgical candidates. ${ }^{4-9}$ Moreover, longitudinal relaxation rate, transverse relaxation rate $\left(\mathrm{R}_{1}, \mathrm{R}_{2}\right)$, and $\mathrm{rCBV}$ decreased as the distance from the contrast enhancement portion of the tumor increased. There was a significant increase in the $\mathrm{R}_{1}$ gradient after contrast agent injection $(P<0.0001)$. There was a heterogeneous pattern of relaxation values in the peritumoral edema adjacent to the contrast enhancement portion of the tumor. This pattern might reflect an invisible tumor infiltrating into the surrounding tissue. This information might be useful for the planning of surgery and radiotherapy. ${ }^{8}$ In addition to $\mathrm{rCBV}$, diffusion kurtosis imaging (DKI) and neurite orientation dispersion and density imaging (NODDI) scalar markers could be used effectively as glioma grade biomarkers and had a significant difference $(P<0.05)$ for grading between low-grade and high-grade gliomas, especially for glioma II vs glioma III and glioma III vs glioma IV. ${ }^{5}$ According to the abovementioned reports, MRI is a very good method for the clinical diagnosis of glioma.

In MRI, MRI contrast agents can enhance the relaxation rates of water molecules and can therefore be used to provide additional information in the image relative to the areas that have not been treated with the agent. ${ }^{10}$ Currently, gadolinium(III)-based contrast agents have been used for $>40 \%$ MRI scans in clinical practice and play an important role in angiography-enhanced MRI studies. ${ }^{11-13}$ There are several MRI contrast agents that have been successfully used for the clinical diagnosis of specific diseases, including Dotarem (Gd-DOTA), ProHance (Gd-HP-DO3A), and Gadovist (Gd-DO3A-burol). ${ }^{14,15}$ Gd-DOTA, a complex prepared with the chelating agent 1,4,7,10-tetraazacyclododecane-1,4,7,10tetraacetic acid (DOTA), exhibits the fastest rate of bound water. ${ }^{16}$ Therefore, many studies currently use Gd-DOTA as a target for drug synthesis studies. ${ }^{17-23,40-42}$

Theranostics, which combines diagnostic and therapeutic effects, is a promising method of personalized medicine, especially for cancer. ${ }^{24,25}$ On the basis of the advantage of permeating the BBB with HDMTX, Gd-DOTA was conjugated to MTX. The fabricated drug, MTX-Gd, would have a broad impact on cancer diagnosis and treatment.

\section{Materials and methods Materials}

All chemicals were obtained from commercial suppliers and were used as received. MTX was purchased from SigmaAldrich Co. (St Louis, MO, USA). Dimethyl sulfoxide (DMSO) was purchased from Shanghai Chemical Co. Ltd, Shanghai, PR China. All solutions were of analytical grade in this study. The C6 glioma cell line was purchased from the China Center for Type Culture Collection (Wuhan University, Wuhan, PR China) and cultured in DMEM (Beijing Dingguo Changsheng Biotechnology Co. Ltd, Beijing, PR China), supplemented with $10 \% \mathrm{FBS}$ and $1 \%$ antibiotics (100 $\mathrm{U} / \mathrm{mL}$ penicillin and $100 \mathrm{mg} / \mathrm{mL}$ streptomycin) at $37^{\circ} \mathrm{C}$ in a $5 \% \mathrm{CO}_{2}$ atmosphere.

\section{Synthesis and characterization of MTX-Gd}

Tri-tert-butyl-2,2',2"-(10-(2-ethoxy-2-oxoethyl)-1,4,7,10tetraazacyclododecane-1,4,7-triyl) triacetate (Tris-DOTA) (1.447 g, $2.4 \mathrm{mmol})$ and MTX (2.3 g, $4.8 \mathrm{mmol})$ were dissolved in $N, N$-dimethylformamide (DMF), placed in a bottle for mixing, and stirred at room temperature for 48 hours. After that, the resulting mixture was evaporated to obtain a light yellow solid. Then, the solid mixture was dissolved in $22 \mathrm{~mL} \mathrm{HCl}$ aqueous solution (37\%), and the solution was stirred for 30 minutes at room temperature. $\mathrm{HCl}$ and water were removed under vacuum to obtain a light yellow solid. Light-yellow solid was added to $20 \mathrm{~mL}$ acetone, and the suspension was stirred for 1 hour at room temperature. The white precipitates were filtered and washed with $10 \mathrm{~mL}$ acetone three times. The solvent was removed under reduced pressure to gain the product MTX-DOTA as a light yellow powder. 
MTX-Gd was prepared according to the reported procedure in the literature. ${ }^{18} \mathrm{In}$ short, the $10 \mathrm{~mL}$ DMF solution without metal MTX-DOTA (346 mg, $0.3 \mathrm{mmol}$ ) and gadolinium acetate hydrate $(352 \mathrm{mg}, 1 \mathrm{mmol})$ was stirred for 24 hours at $50^{\circ} \mathrm{C}$. The solvent was removed and dissolved in water. The precipitates were filtered and washed with acetone three times and then purified by alumina column chromatography (toluene acetone-DMSO as eluent, DMSO/ $\mathrm{H}_{2} \mathrm{O}=1 / 0-8 / 2$ fraction).

\section{In vitro cytotoxicity assay by the MTT assay}

C6 cells were plated in 96-well plates at $5.0 \times 10^{4}$ cells/well and cultured in $100 \mu \mathrm{L} \mathrm{DMEM}$ with $10 \% \mathrm{FBS}$ at $37^{\circ} \mathrm{C}$ in $5 \% \mathrm{CO}_{2}$ atmosphere for 24 hours. Subsequently, the cells were incubated under concentrations of $0.04-270 \mu \mathrm{M}$ of MTX or MTX-Gd for 48 hours. After incubation, the media were removed, and the cells were washed with PBS. To each well, $20 \mu \mathrm{L}$ MTT solution ( $5 \mathrm{mg} / \mathrm{mL}$ in PBS) and $80 \mu \mathrm{L}$ DMEM were added and incubated for 3-4 hours. The media were then completely removed, and $150 \mu \mathrm{L}$ of DMSO was added to each well; as a result, the color changed from yellow to blue. The absorbance of the solution was measured using a microplate reader at $450 \mathrm{~nm}$. The degree of cell viability in each sample was calculated by the following formula: cell viability $(\%)=\mathrm{A}_{\text {sample }} / \mathrm{A}_{\text {control }} \times 100 \%$, where $\mathrm{A}_{\text {sample }}$ and $\mathrm{A}_{\text {control }}$ are the absorbance values for the treated cells and the untreated control cells, respectively. The $\mathrm{A}_{\text {sample }}$ and $\mathrm{A}_{\text {control }}$ values were obtained after subtracting the absorbance of DMSO. Data are presented as the average $\pm S D(n=4)$.

\section{TI-weighted and TI-mapping images of MTX-Gd in vitro and in vivo}

All animals received care in compliance with the guidelines outlined in the Guide for the Care and Use of Laboratory Animals, and the procedures were approved by the Wuhan University of China Animal Care and Use Committee. Female BALB/c mice were obtained from Beijing Huafukang Bioscience Co. Ltd, Beijing, PR China. with weights of 14-16 $\mathrm{g}$ and provided and maintained with free access to food and water.

The T1-weighted and T1-mapping images of MTX-Gd were measured at $37^{\circ} \mathrm{C}$ using the Siemens Prisma 3T MRI scanner. MTX-Gd was dispersed in double-distilled $\mathrm{H}_{2} \mathrm{O}$ $\left(\mathrm{ddH}_{2} \mathrm{O}\right)$ at different concentrations. For MRI measurements, each of the 96-well plates was filled with $150 \mu \mathrm{L}$ solution to achieve T1-weighted and T1-mapping images. For in vivo MRI, tumor-bearing BALB/c nude mice were injected with $200 \mu \mathrm{L}$ of MTX-Gd solution $(2 \mathrm{mM})$ through the tail vein. Then, the mice were scanned using the Siemens Prisma 3T MRI scanner at 30 minutes, 1 hour, 2 hours, and 3 hours after the injection. The T1-weighted image parameters were described as follows: echo time, $12 \mathrm{~ms}$; repetition time, 700 $\mathrm{ms}$; field of view, $120 \times 120 \mathrm{~mm}$; matrix size, $400 \times 400$; slice thickness, $2.0 \mathrm{~mm}$; and the number of acquisitions, 14 . The T1 mapping parameters were described as follows: repetition time, $15 \mathrm{~ms}$; echo time, $2.7 \mathrm{~ms}$; field of view, $160 \times 160$ $\mathrm{mm}$; image size, $768 \times 768$; slice thickness, $2.0 \mathrm{~mm}$; and the number of acquisitions, 14 . The T1-mapping value was measured three times at each MTX-Gd concentration, and the mean of the $\mathrm{T} 1$ values was recorded. Taking the longitudinal relaxation time $\left(r_{1}\right)$ as the abscissa and the inverse longitudinal relaxation time $(1 / \mathrm{T} 1)$ as the ordinate, a fitting curve was made to represent the MTX-Gd function. The control groups of MTX or Gd-DOTA also used the abovementioned method for data collection.

\section{Results and discussion Synthesis and characterization of MTX-Gd} MTX-Gd was prepared as shown in Figure 1, and MTX-Gd was synthesized by connecting MTX and Gd through DOTA. To detect whether the MTX-Gd was synthesized successfully, it was detected by ultraviolet (UV) and infrared radiation (IR) spectroscopy. The ultraviolet-visible-near infrared radiation (UV-NIR) absorption curve indicated that MTX-Gd had a broad absorption in the NIR region $(500-700 \mathrm{~nm}$; Figure 2A). The formation of MTX-Gd was confirmed from the characteristic bands of MTX-DOTA-Gd in the $1,413 \mathrm{~cm}^{-1}$ $(\mathrm{C}-\mathrm{N}), 1,577 \mathrm{~cm}^{-1}\left(-\mathrm{NH}_{2}\right)$, and $3,429 \mathrm{~cm}^{-1}(\mathrm{~N}-\mathrm{H})$, in the Fourier transform IR (FTIR) spectra (Figure 2B).

\section{In vitro cytotoxicity analysis by the MTT assay}

To confirm the antitumor effect of MTX-Gd, the cytotoxicity of MTX-Gd was examined by the MTT assay. C6 cells were cultured in 96-well plates. At 24 hours after incubation, MTX-Gd or MTX was added to the cells, and the complex was incubated for 48 hours. Subsequently, the absorbance of the solution was measured using a microplate reader at $450 \mathrm{~nm}$. Figure 3 shows that the MTX-Gd showed little difference in the cell viability compared with the control group, except for the highest concentration. The cytotoxicity experiments demonstrated that the synthesis of MTX-Gd had the 
<smiles>CC(C)CC(=O)CN1CCN(CC(=O)O)CCN(CC(=O)CC(C)C)CN(CC(=O)CC(C)C)CC1</smiles>

Tris(tert-butyl ester)-DOTA<smiles>CN(Cc1cnc2nc(N)nc(N)c2n1)c1ccc(C(=O)NC(CCC(=O)O)C(=O)O)cc1</smiles>

MTX<smiles>CC(C)CC(=O)CN(CCN(CCC(=O)Nc1nc(N)c2nc(CN(C)c3ccc(C(=O)NC(CCC(=O)O)C(=O)O)cc3)cnc2n1)CC(=O)O)CCN(CC(=O)CC(C)C)CC(=O)CC(C)C</smiles>

Tris(tert-butyl ester)-DOTA-MTX<smiles>Cc1nc(NC(=O)CN(CCN(CCN(CC(=O)O)CC(=O)O)CCN(CC(=O)O)CC(C)(Cl)Cl)CC(=O)O)nc2ncc(CN(C)c3ccc(C(=O)NC(CCC(=O)O)C(=O)O)cc3)nc12</smiles>

MTX-DOTA-Gd

Figure I The synthesis pathway of MTX-Gd.

Abbreviations: DMF, N,N-dimethylformamide; DOTA, I,4,7,I0-tetraazacyclododecane-I,4,7,I0-tetraacetic acid; MTX, methotrexate; EDC, I-ethyl-3-(3-dimethylaminopropyl)carbodiimide hydrochloride; NHS, N-hydroxysuccinimide; Gd, gadolinium(III); $\mathrm{Gd}(\mathrm{AC})_{3}$, $\mathrm{Gd}(\mathrm{CH} 3 \mathrm{COOH})_{3}$.

same treatment effect as MTX at the same dose. However, the reason why MTX-Gd had more cytotoxicity than MTX at the highest concentration still needs to be explored. In addition, it is also revealed that the MTX-Gd conjugates do not lose the function of MTX after they are linked to DOTA-Gd.
Furthermore, a previous study reported that Gd-DOTA is generally not toxic to normal cells at low concentrations. The data showed that it had cytotoxicity to normal cells when the concentration of Gd-DOTA was above $400 \mu \mathrm{M}$, otherwise it showed no toxicity. ${ }^{26}$

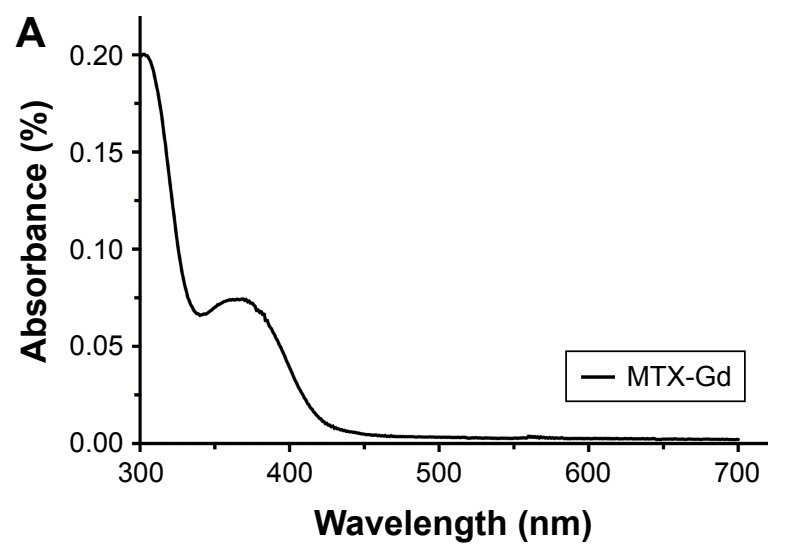

B

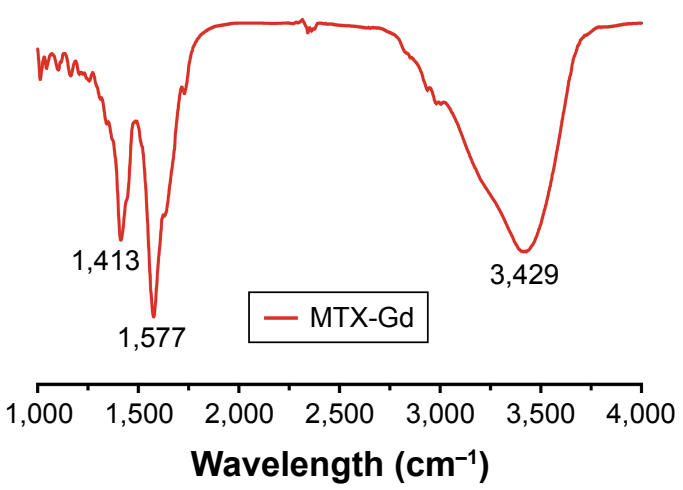

Figure 2 (A) UV-visible-NIR absorbance spectra of MTX-Gd. (B) FTIR spectra of MTX-Gd.

Abbreviations: FTIR, Fourier-transform infrared; MTX, methotrexate; NIR, near infrared; UV, ultraviolet; Gd, gadolinium(III). 

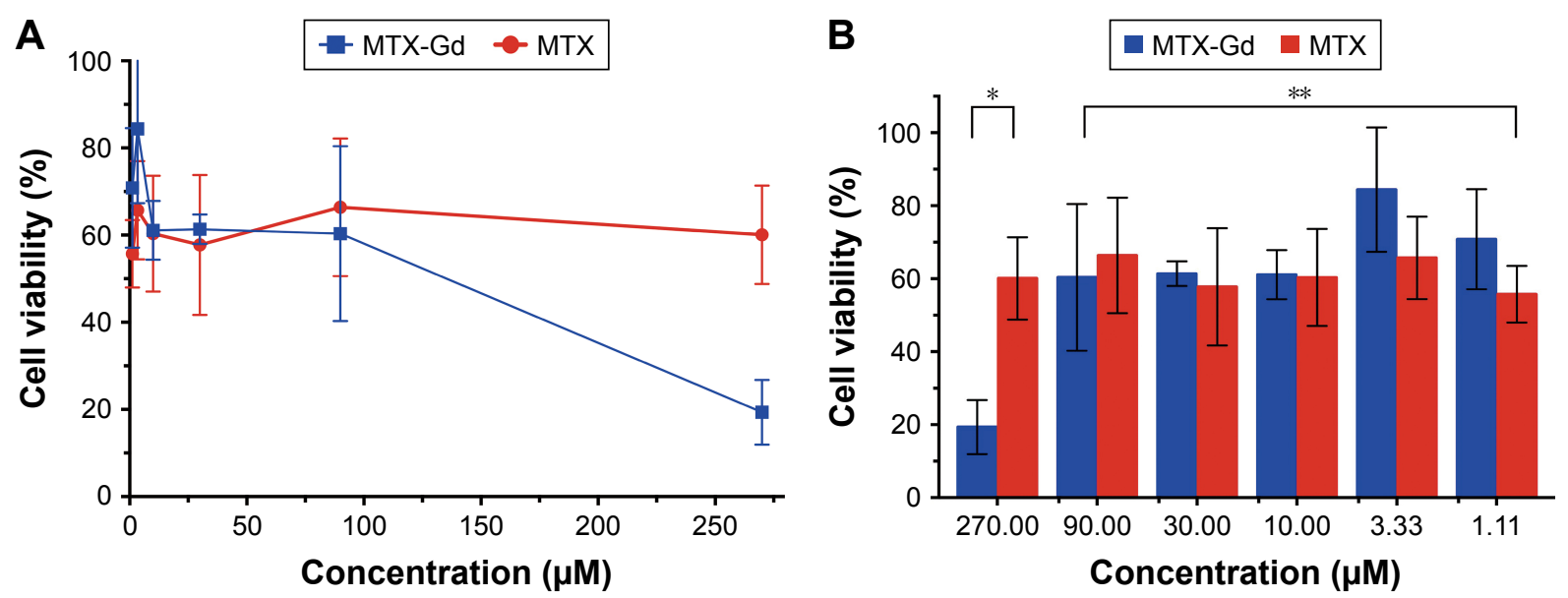

Figure 3 After treating the cells with various concentrations of complexes for 48 hours, the cytotoxic effects of MTX-Gd and MTX were detected using the MTT assay. Notes: Data represent the average of two independent experiments. (A) The curve indicates the cell viability of different concentrations of MTX-Gd or MTX. (B) Histogram indicates the cell viability of different concentrations of MTX-Gd or MTX. $* P<0.05$, for all comparisons, indicates a difference in cell viability. $* * P>0.05$, for all comparisons, indicates no difference in cell viability.

Abbreviations: MTX, methotrexate; Gd, gadolinium(III).

A

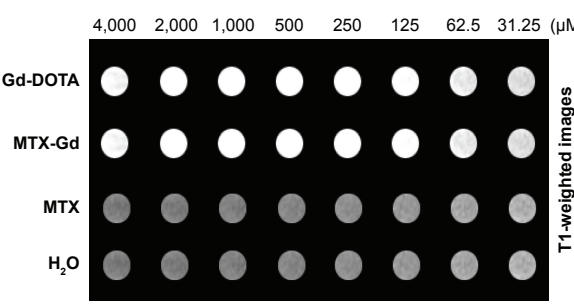

B

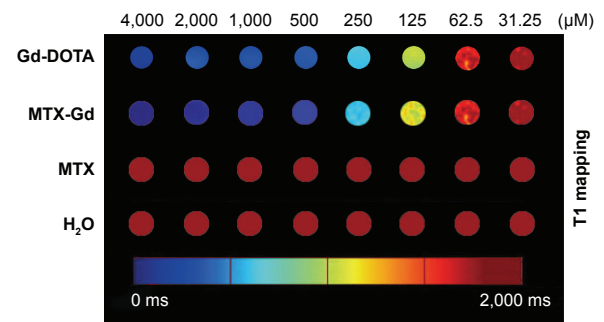

$\mathbf{E}$

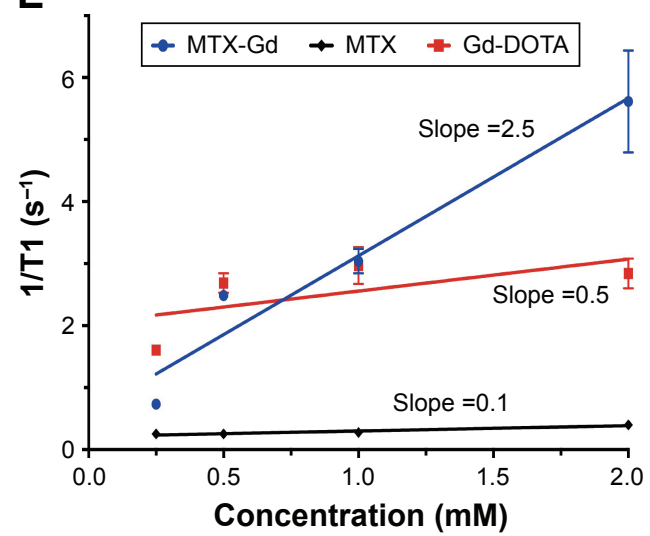

C

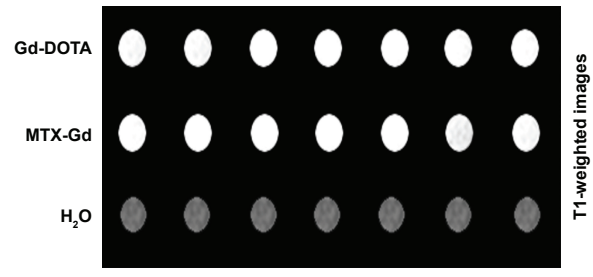

D

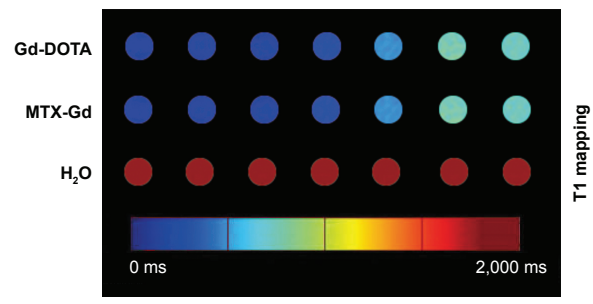

$\mathbf{F}$

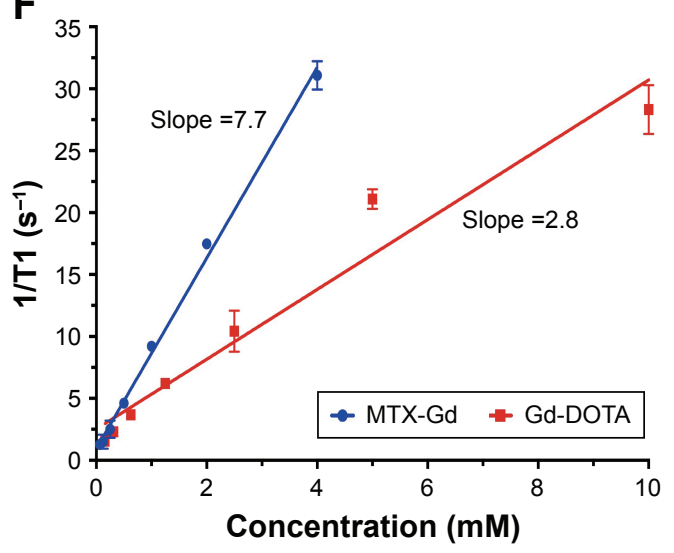

Figure 4 The signal intensity of MTX-Gd, MTX, and Gd-DOTA was measured by MRI in vitro.

Notes: MTX-Gd, MTX, or Gd-DOTA was measured by (A) TI-weighted images and (B) TI-mapping images. In vitro (C) TI-weighted images and (D) TI-mapping images of MTX-Gd at different concentrations (4,000, 2,000, I,000, 500, 250, I25, and $62.5 \mu \mathrm{M})$. In vitro (C) TI-weighted images and (D) TI-mapping images of Gd-DOTA at different concentrations (10,000, 5,000, 2,500, I,250, 625, 312.5, and I56.25 $\mu \mathrm{M})$. (E) The curve indicates the gradient concentration with maximum concentration of $2 \mathrm{mM}$ of MTX-Gd, MTX, or Gd-DOTA. (F) The blue line represents the gradient concentration with a maximum concentration of 4 mM of MTX-Gd. The red line represents the gradient concentration with a maximum concentration of $10 \mathrm{mM}$ of Gd-DOTA.

Abbreviations: DOTA, I,4,7, I0-tetraazacyclododecane-I,4,7, I0-tetraacetic acid; MRI, magnetic resonance imaging; MTX, methotrexate; Gd, gadolinium(III). 
TI-weighted and TI-mapping images of MTX-Gd in vitro and in vivo

To investigate the contrast enhancement of the MTX-Gd, MRI was measured in vitro. As expected, due to the paramagnetic relaxivity enhancement (PRE) effect, MTX-Gd showed high proton relaxivity $\left(\mathrm{r}_{1}\right)$ in $\mathrm{ddH}_{2} \mathrm{O}$ under the $3 \mathrm{~T}$ MRI system (Figure 4). As shown in Figure 4B, the imaging of MTX-Gd was significantly brighter than the control groups at the same concentration, and the brightness and signal intensity of MRI were increased followed by the increased concentration of MTX-Gd. In addition, it also showed that MTX was not visualized on MRI. In Figure 4D, the images revealed that the concentration of $4 \mathrm{mM}$ MTX-Gd had the same imaging effect with the concentration of $10 \mathrm{mM}$ Gd-DOTA. The images demonstrated that MTX-Gd at the low concentration could obtain a high signal intensity, while Gd-DOTA needed a high concentration. Moreover, as a key parameter of the contrast agent, $r_{1}$ was obtained from the slope of the fit linear of the
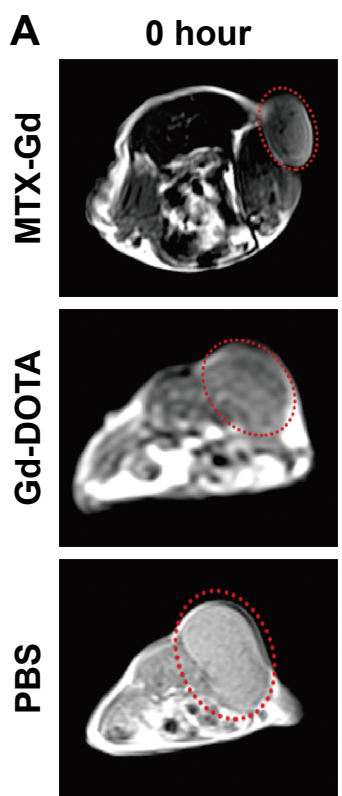

B
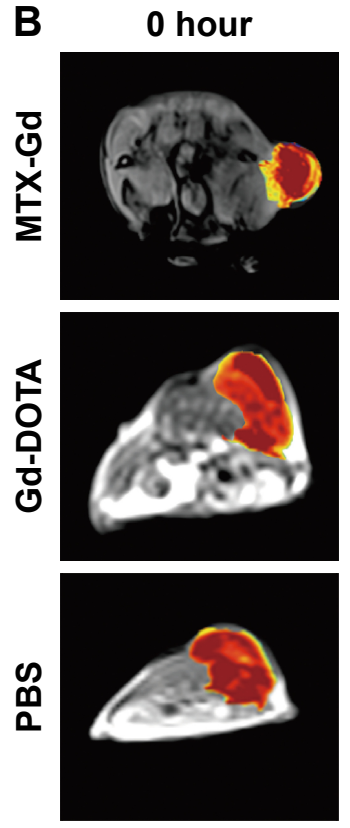

0.5 hour
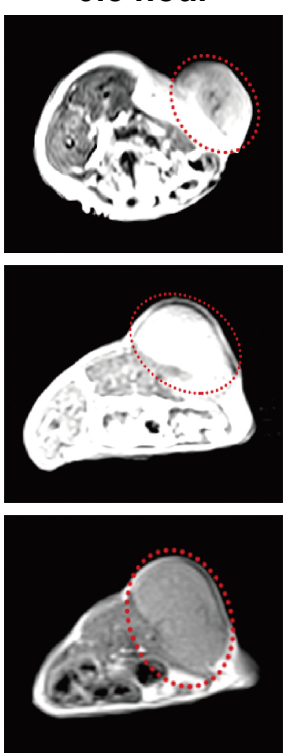

0.5 hour
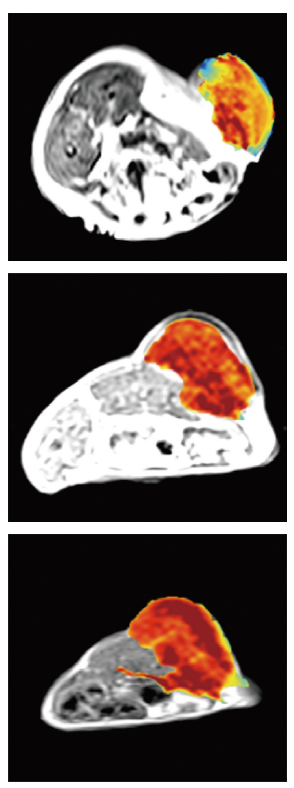

1 hour
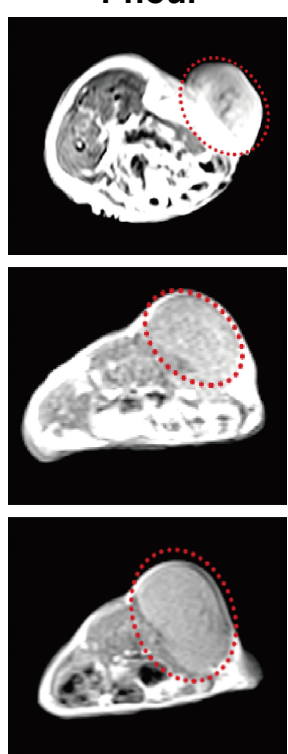

1 hour
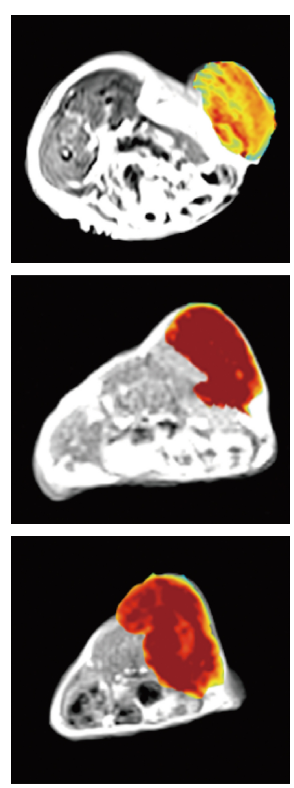

2 hours
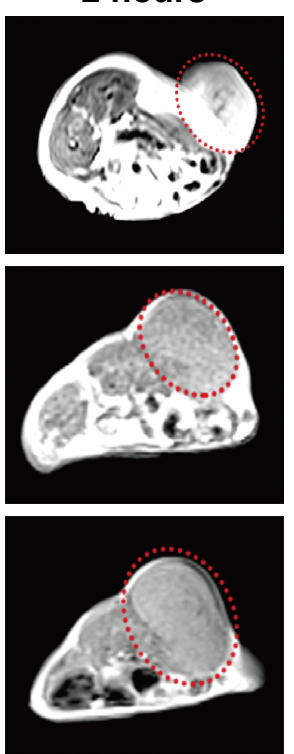

2 hours
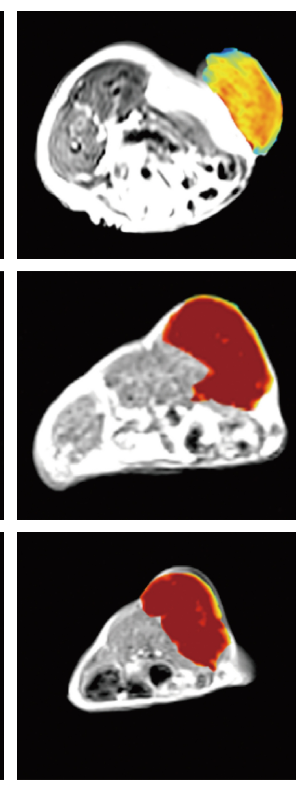

3 hours
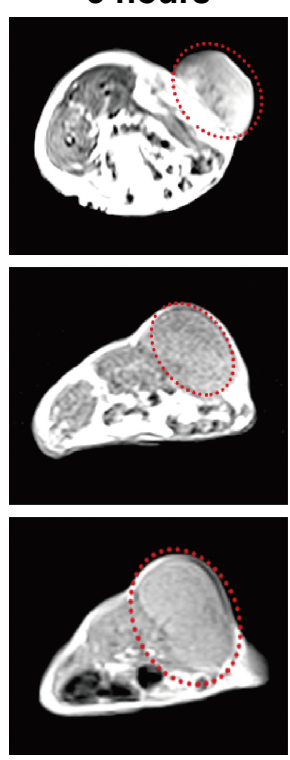

3 hours
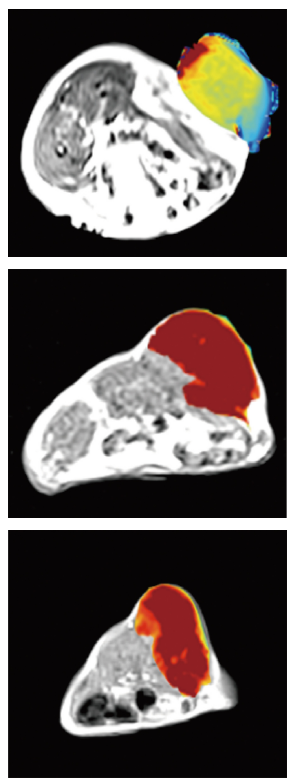


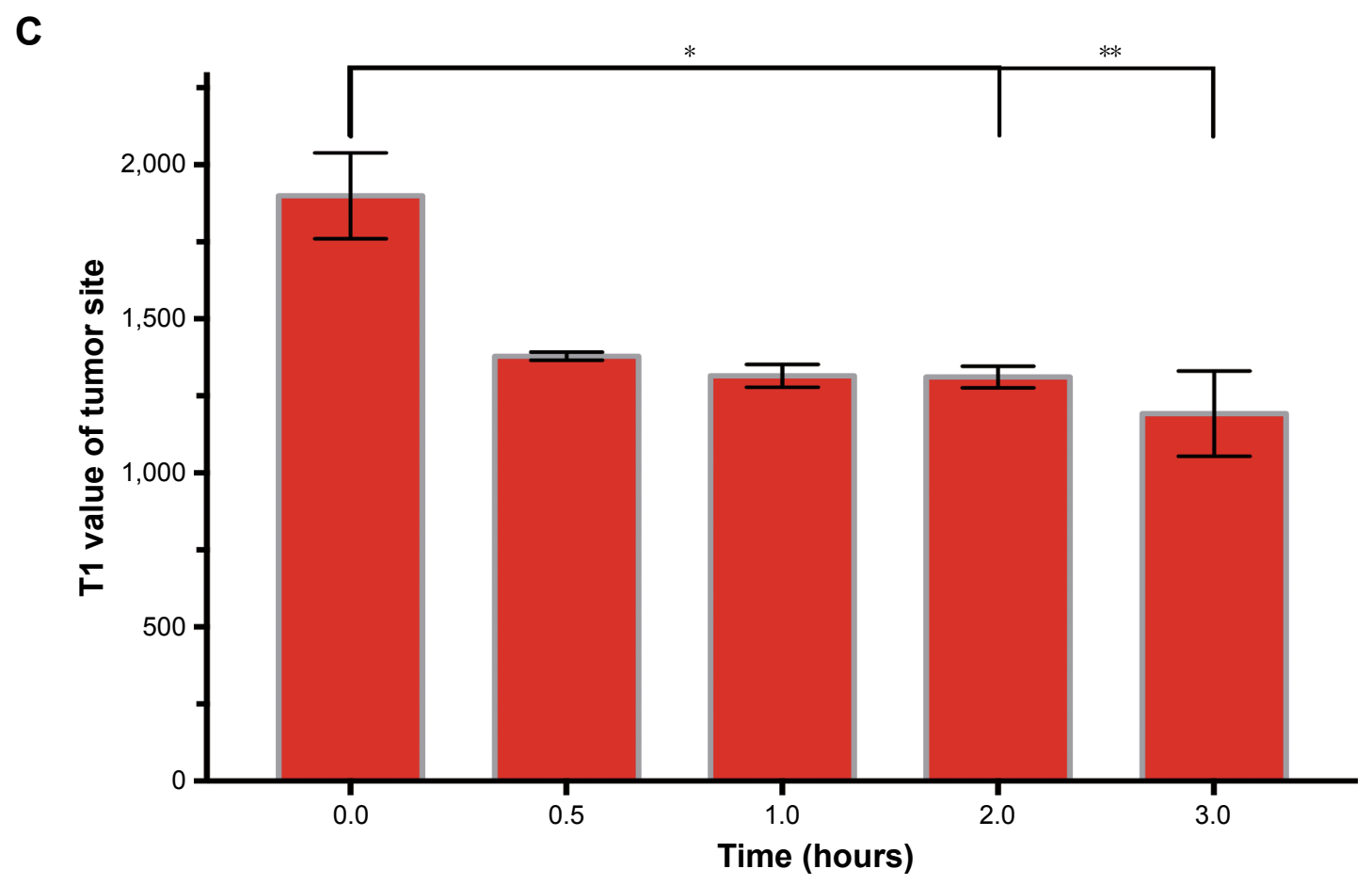

Figure 5 (A) TI-weighted images and (B) the corresponding mapping images of tumors after injection of MTX-Gd, Gd-DOTA, and PBS at different time points. (C) TI-mapping values of tumors were measured after different injection times of MTX-Gd $(* P<0.05$, $* * P<0.005)$.

Abbreviations: DOTA, I,4,7, I0-tetraazacyclododecane- I,4,7,10-tetraacetic acid; MTX, methotrexate; Gd, gadolinium(III).

reciprocal of the $\mathrm{T} 1$ relaxation time. As shown in Figure 4F, when MTX-Gd and Gd-DOTA had the same signal intensity, the $r_{1}$ of MTX-Gd was $7.7 \mathrm{mM}^{-1} \mathrm{~s}^{-1}$, while the $\mathrm{r}_{1}$ of the commercial imaging agent Gd-DOTA was $2.8 \mathrm{mM}^{-1} \mathrm{~s}^{-1}$. Then, nude mice bearing C6 tumor xenografts were used to study in vivo imaging capabilities. After the tail vein injection of MTX-Gd, significant contrast enhancement was observed at the tumor site from 0.5 to 3 hours (Figure 5); the signal of tumor area was strongest at 3 hours due to accumulation by the size effect of macromolecules. This finding provided a longer viewing window than the clinic contrast agent such as Gd-DOTA, which will rapidly metabolize within 30 minutes. The results revealed that MTX-Gd had a better MRI contrast effect than Gd-DOTA, which further suggests that MTX-Gd has a good MRI contrast characteristic.

\section{Conclusion}

A novel stable and unique theranostic agent (MTX-Gd) was successfully synthesized, which was specifically designed for cancer diagnosis and treatment. The data showed that MTX-Gd has good stability, strong anticancer ability, and excellent magnetic capacity. The MTX component of MTX-Gd, as a chemotherapeutic agent, played an important role in targeted therapies of cancer. The DOTA-Gd component of MTX-Gd performed as the MRI contrast agent.
The superior MRI performance and synergetic chemical antineoplastic ability of MTX-Gd were revealed, and it has great potential in the diagnosis and treatment of gliomas and potentially other cancers, with prospects of clinical application in the near future.

\section{Acknowledgments}

This work was financially supported by the National Natural Science Foundation of China (81701685, 81771819), the National Key Research and Development Project (2017YFC0108803), the Fundamental Research Funds for the Central Universities (2042018kf0245), and Zhongnan Hospital of Wuhan University Science, Technology and Innovation Seed Fund (Project cxpy20160058, cxpy20160011).

\section{Disclosure}

The authors report no conflicts of interest in this work.

\section{References}

1. Stone JB, Deangelis LM. Cancer-treatment-induced neurotoxicity-focus on newer treatments. Nat Rev Clin Oncol. 2016;13(2):92-105.

2. Joerger M, Huitema AD, Illerhaus G, Ferreri AJ. Rational administration schedule for high-dose methotrexate in patients with primary central nervous system lymphoma. Leuk Lymphoma. 2012;53(10):1867-1875.

3. Angelov L, Doolittle ND, Kraemer DF, et al. Blood-brain barrier disruption and intra-arterial methotrexate-based therapy for newly diagnosed primary CNS lymphoma: a multi-institutional experience. J Clin Oncol. 2009;27(21):3503-3509. 
4. Soliman RK, Gamal SA, Essa AA, Othman MH. Preoperative Grading of Glioma Using Dynamic Susceptibility Contrast MRI: Relative Cerebral Blood Volume Analysis of Intra-tumoural and Peri-tumoural Tissue. Clin Neurol Neurosurg. 2018;167:86-92.

5. Maximov II, Tonoyan AS, Pronin IN. Differentiation of glioma malignancy grade using diffusion MRI. Phys Med. 2017;40:24-32.

6. Narang AK, Chaichana KL, Weingart JD, et al. Progressive LowGrade Glioma: Assessment of Prognostic Importance of Histologic Reassessment and MRI Findings. World Neurosurg. 2017;99: 751-757.

7. Firouznia S, Sabetrasekh P, Ahmadian A. Multimodal MRI Techniques and Their Importance in Frameless Navigation Biopsy for Brain Glioma. Iran J Radiol. 2017;14(4):e65721.

8. Blystad I, Warntjes JBM, Smedby Ö, Lundberg P, Larsson EM, Tisell A. Quantitative MRI for analysis of peritumoral edema in malignant gliomas. PLoS One. 2017;12(5):e0177135.

9. Leu K, Ott GA, Lai A, et al. Perfusion and diffusion MRI signatures in histologic and genetic subtypes of WHO grade II-III diffuse gliomas. J Neurooncol. 2017;134(1):177-188.

10. Chan K, Wong W. Small molecular gadolinium(III) complexes as MRI contrast agents for diagnostic imaging. Coord Chem Rev. 2007; 251(17-20):2428-2451.

11. Werner EJ, Datta A, Jocher CJ, Raymond KN. High-relaxivity MRI contrast agents: where coordination chemistry meets medical imaging. Angew Chem Int Ed Engl. 2008;47(45):8568-8580.

12. Major JL, Meade TJ. Bioresponsive, cell-penetrating, and multimeric MR contrast agents. Acc Chem Res. 2009;42(7):893-903.

13. Cheng W, Haedicke IE, Nofiele J, et al. Complementary strategies for developing Gd-free high-field $\mathrm{T}_{1}$ MRI contrast agents based on Mn(III) porphyrins. J Med Chem. 2014;57(2):516-520.

14. Chong HS, Song HA, Lim S, et al. A novel cholic acid-based contrast enhancement agent for targeted MRI. Bioorg Med Chem Lett. 2008; 18(7):2505-2508.

15. Li Y, Liu G, Lou X, Chen Z, Ma L. Intra-individual comparison of different gadolinium-based contrast agents in the quantitative evaluation of C6 glioma with dynamic contrast-enhanced magnetic resonance imaging. Sci China Life Sci. 2017;60(1):11-15.

16. Aydın Tekdaş D, Garifullin R, Şentürk B, et al. Design of a GdDOTA-phthalocyanine conjugate combining MRI contrast imaging and photosensitization properties as a potential molecular theranostic. Photochem Photobiol. 2014;90(6):1376-1386.

17. Wu B, Lu ST, Zhang LJ, Zhuo RX, Xu HB, Huang SW. Codelivery of doxorubicin and triptolide with reduction-sensitive lipid-polymer hybrid nanoparticles for in vitro and in vivo synergistic cancer treatment. Int J Nanomedicine. 2017;12:1853-1862.

18. Wu B, Li XQ, Huang T, et al. MRI-guided tumor chemo-photodynamic therapy with $\mathrm{Gd} / \mathrm{Pt}$ bifunctionalized porphyrin. Biomater Sci. 2017; 5(9):1746-1750.

19. Wu B, Wan B, Lu ST, et al. Near-infrared light-triggered theranostics for tumor-specific enhanced multimodal imaging and photothermal therapy. Int J Nanomedicine. 2017;12:4467-4478.

20. Tan M, Ye Z, Lindner D, Brady-Kalnay SM, Lu ZR. Synthesis and evaluation of a targeted nanoglobular dual-modal imaging agent for MR imaging and image-guided surgery of prostate cancer. Pharm Res. 2014;31(6):1469-1476.

21. Zhou Z, Wu X, Kresak A, Griswold M, Lu ZR. Peptide targeted tripod macrocyclic Gd(III) chelates for cancer molecular MRI. Biomaterials. 2013;34(31):7683-7693.

22. Zhang P, Zhang Y, Li B, et al. Cell-assembled nanoclusters of MSCtargeting Gd-DOTA-peptide as a $\mathrm{T}_{2}$ contrast agent for MRI cell tracking. J Pept Sci. 2018;24(4-5):e3077.

23. Wang H, Sun D, Liao H, et al. Synthesis and characterization of a bimodal nanoparticle based on the host-guest self-assembly for targeted cellular imaging. Talanta. 2017;171:8-15.
24. Lammers T, Aime S, Hennink WE, Storm G, Kiessling F. Theranostic nanomedicine. Acc Chem Res. 2011;44(10):1029-1038.

25. Kelkar SS, Reineke TM. Theranostics: combining imaging and therapy. Bioconjug Chem. 2011;22(10):1879-1903.

26. Zhang J, Li Y, Zhang Q, Hao X, Wang S. Effects of gadolinium on proliferation, differentiation and calcification of primary mouse osteoblasts in vitro. J Rare Earths. 2012;30(8):831-834.

27. Abrey LE, Moskowitz CH, Mason WP, et al. Intensive methotrexate and cytarabine followed by high-dose chemotherapy with autologous stemcell rescue in patients with newly diagnosed primary CNS lymphoma: an intent-to-treat analysis. J Clin Oncol. 2003;21(22):4151-4156.

28. Batchelor T, Carson K, O’Neill A, et al. Treatment of primary CNS lymphoma with methotrexate and deferred radiotherapy: a report of NABTT 96-07. J Clin Oncol. 2003;21(6):1044-1049.

29. Choi YJ, Park H, Lee JS, et al. Methotrexate elimination and toxicity: MTHFR $677 \mathrm{C}>\mathrm{T}$ polymorphism in patients with primary CNS lymphoma treated with high-dose methotrexate. Hematol Oncol. 2017; 35(4):504-509.

30. Fritsch K, Kasenda B, Schorb E, et al. High-dose methotrexate-based immuno-chemotherapy for elderly primary CNS lymphoma patients (PRIMAIN study). Leukemia. 2017;31(4):846-852.

31. Hoang-Xuan K, Bessell E, Bromberg J, et al. Diagnosis and treatment of primary CNS lymphoma in immunocompetent patients: guidelines from the European Association for Neuro-Oncology. Lancet Oncol. 2015; 16(7):e322-e332.

32. Okita Y, Narita Y, Miyakita Y, et al. Health-related quality of life in outpatients with primary central nervous system lymphoma after radiotherapy and high-dose methotrexate chemotherapy. Mol Clin Oncol. 2016;5(3):179-185.

33. Weller M, Wick W, Aldape K, et al. Glioma. Nat Rev Dis Primers. 2015; 1:15017.

34. Esmaeili M, Stensjøen AL, Berntsen EM, Solheim O, Reinertsen I. The Direction of Tumour Growth in Glioblastoma Patients. Sci Rep. 2018;8(1):1199.

35. Schuenke P, Paech D, Koehler C, et al. Fast and Quantitative T1 1 weighted Dynamic Glucose Enhanced MRI. Sci Rep. 2017;7:42093.

36. Xuesong D, Wei X, Heng L, et al. Evaluation of neovascularization patterns in an orthotopic rat glioma model with dynamic contrast-enhanced MRI. Acta Radiol. 2017;58(9):1138-1146.

37. Khodadadei F, Safarian S, Ghanbari N. Methotrexate-loaded nitrogendoped graphene quantum dots nanocarriers as an efficient anticancer drug delivery system. Mater Sci Eng C Mater Biol Appl. 2017;79: 280-285.

38. Vakilinezhad MA, Alipour S, Montaseri H. Fabrication and in vitro evaluation of magnetic PLGA nanoparticles as a potential Methotrexate delivery system for breast cancer. J Drug Deliv Sci Technol. 2018;44: 467-474.

39. Maestá I, Nitecki R, Horowitz NS, et al. Effectiveness and toxicity of first-line methotrexate chemotherapy in low-risk postmolar gestational trophoblastic neoplasia: The New England Trophoblastic Disease Center experience. Gynecol Oncol. 2018;148(1):161-167.

40. Park JA, Lee YJ, Ko IO, Io K, et al. Improved tumor-targeting MRI contrast agents: Gd(DOTA) conjugates of a cycloalkane-based RGD peptide. Biochem Biophys Res Commun. 2014;455(3-4):246-250.

41. Xiao Y, Xue R, You T, et al. Gadolinium-1,4,7,10-tetraazacyclododecane1,4,7,10-tetraacetic acid conjugate of arabinogalactan as a potential liver-targeting magnetic resonance imaging contrast agent. Carbohydr Res. 2014;395:9-14.

42. Li Y, Han Z, Roelle S, et al. Synthesis and Assessment of Peptide GdDOTA Conjugates Targeting Extradomain B Fibronectin for Magnetic Resonance Molecular Imaging of Prostate Cancer. Mol Pharm. 2017; 14(11):3906-3915. 


\section{Publish your work in this journal}

Drug Design, Development and Therapy is an international, peerreviewed open-access journal that spans the spectrum of drug design and development through to clinical applications. Clinical outcomes, patient safety, and programs for the development and effective, safe, and sustained use of medicines are the features of the journal, which has also been accepted for indexing on PubMed Central. The manuscript management system is completely online and includes a very quick and fair peer-review system, which is all easy to use. Visit http://www.dovepress.com/testimonials.php to read real quotes from published authors.

Submit your manuscript here: http://www.dovepress.com/drug-design-development-and-therapy-journal 